\title{
ARTÍCULO ORIGINAL \\ Tendencias de la investigación sobre síntomas de trastornos mentales durante la pandemia por COVID-19
}

Fecha de recibido:

25 de mayo de 2020

Fecha de aprobación:

23 de junio de 2020
Forma de citar este artículo: Martínez JA, Bolívar Y, Yanez-Peñúñuri LY, Rey CA. Tendencias de la investigación sobre síntomas de trastornos mentales durante la pandemia por COVID-19. Med UPB. 2020;39(2):24-33. DOI:10.18566/medupb.v39n2.a05

1 Grupo de Investigación en Psicología Clínica y de la Salud, Universidad Pedagógica y

Tecnológica de Colombia. Tunja, Colombia.

2 Grupo de Investigación Municipio Saludable por la Paz, Universidad Pedagógica y Tecnológica de Colombia. Tunja, Colombia.

3 Universidad de Sonora. Sonora, México..

\section{Dirección de}

correspondencia: Jorge Arturo Martínez Gómez. Correo electrónico: jorgearturo.martinez@uptc. edu.co

\section{Research tendencies about mental disorder symptoms during the COVID-19 pandemic / Tendências da investigação sobre sintomas de transtornos mentais durante a pandemia por COVID-19}

Jorge Arturo Martínez Gómez¹, Yolima Bolívar Suárez², Libia Yanelli Yanez-Peñúñuri³, César Armando Rey Anacona ${ }^{1}$

\section{RESUMEN}

Objetivo: Realizar una revisión sistemática de la tendencia de investigación sobre síntomas de trastornos mentales durante la pandemia por COVID-19.

Métodos: Se recuperaron artículos publicados entre el 31 de diciembre 2019 y el 3 de mayo 2020, en Pubmed, SCOPUS, EBSCO, PsycINFO, Science Direct, Redalyc y Web of Science, seleccionando, conforme a la metodología PRISMA, un total de 16 artículos, al descartarse duplicados o estudios no empíricos.

Resultados: La mayoría se realizaron en China, el tamaño de la muestra osciló entre 20 y 7236 participantes, en su mayoría adolescentes y adultos, entre 12 a 80 años, con muestreo no probabilístico por conveniencia. La tendencia de los síntomas y trastornos más estudiados fueron ansiedad (28\%), depresión (23\%), estrés (15.3\%), trastorno del sueño-vigilia (9.6\%) y trastorno por estrés postraumático (5.7\%), asociados con el consumo de sustancias psicoactivas, bajo rendimiento académico, pérdida de trabajo, calidad del sueño, bajo autocuidado y baja percepción del estado de salud durante la pandemia. Conclusiones: Los síntomas y trastornos de ansiedad, depresión y estrés son los más frecuentes. Algunas limitaciones son la ausencia de medición previa a la pandemia o cuarentena y el uso de muestreo no aleatorio, para hacer generalización de resultados. Es necesario formular investigaciones de intervención para mitigar el impacto psicológico. Los sistemas de salud deben ofrecer programas de prevención y de promoción de la salud, que consideren factores psicosociales dirigidos a la población general, pacientes y personal sanitario, para disminuir la prevalencia de estas dificultades, en particular, para Iberoamérica, en donde es escasa la producción científica al respecto.

Palabras clave: pandemia; infecciones por Coronavirus; trastorno mental; ansiedad; depresión; estrés; investigación

\section{ABSTRACT}

Objective: To carry out a systematic review of the research trend on mental disorders symptoms during the COVID -19 pandemic.

Methods: Articles published between December 31, 2019 and May 3, 2020 were retrieved in Pubmed, SCOPUS, EBSCO, PsycINFO, Science Direct, Redalyc, and Web of Science, selected according to the PRISMA methodology, a total of 16 articles by ruling out duplicates or non-empirical studies.

Results: Most were carried out in China, the sample size ranged between 20 and 7236 participants, mostly adolescents and adults, between 12 and 80 years old, with non-probability sampling for convenience. The most studied symptoms and disorders 
were anxiety (28\%), depression (23\%), stress (15.3\%), sleep-wake disorder (9.6\%) and post-traumatic stress disorder (5.7\%), associated with psychoactive substance use, low academic performance, job loss, sleep quality, low self-care, and low perception of health status during the pandemic.

Conclusions: The symptoms and disorders of anxiety, depression, and stress are the most frequent. Some limitations are the absence of pre-pandemic or quarantine measurement and the use of non-random sampling to generalize the results. Intervention research needs to be formulated to mitigate the psychological impact. Health systems must offer prevention and health promotion programs that consider psychosocial factors aimed at the general population, patients, and health personnel, to decrease the prevalence of these difficulties, particularly for Latin America where about scientific production is scarce. Keywords: pandemic; Coronavirus infections; mental disorder; anxiety; depression; stress; research

\section{RESUMO}

Objetivo: Realizar uma revisão sistemática da tendência de investigação sobre sintomas de transtornos mentais durante a pandemia por COVID-19.

Métodos: Se recuperaram artigos publicados entre 31 de dezembro 2019 e 3 de maio 2020, em Pubmed, SCOPUS, EBSCO, PsycINFO, Science Direct, Redalyc e Web of Science, selecionando, conforme à metodologia PRISMA, um total de 16 artigos, ao descartar-se duplicados ou estudos não empíricos.

Resultados: A maioria se realizaram na China, o tamanho da amostra oscilou entre 20 e 7236 participantes, na sua maioria adolescentes e adultos, entre 12 a 80 anos, com amostragem não probabilístico por conveniência. A tendência dos sintomas e transtornos mais estudados foram ansiedade (28\%), depressão (23\%), estresse (15.3\%), transtorno do sono-vigília (9.6\%) e transtorno por estresse pós-traumático (5.7\%), associados com o consumo de substâncias psicoativas, sob rendimento acadêmico, perda de trabalho, qualidade do sono, baixo autocuidado e baixa percepção do estado de saúde durante a pandemia. Conclusões: Os sintomas e transtornos de ansiedade, depressão e estresse são os mais frequentes. Algumas limitações são a ausência de medição prévia à pandemia ou quarentena e o uso de amostragem não aleatório, para fazer generalização de resultados. É necessário formular investigações de intervenção para mitigar o impacto psicológico. Os sistemas de saúde devem oferecer programas de prevenção e de promoção da saúde, que considerem fatores psicossociais dirigidos à população geral, pacientes e pessoal sanitário, para diminuir a prevalência destas dificuldades, em particular, para Ibero América, onde é escassa a produção científica ao respeito.

Palavras chave: pandemia; infecções por Coronavírus; transtorno mental; ansiedade; depressão; estresse; investigação.

\section{INTRODUCCIÓN}

Los coronavirus son una extensa familia de virus que causan infecciones respiratorias. E1 SARS-CoV-2 hace parte de esta familia y es el causante de la actual pandemia de COVID-1912, . Los síntomas más comunes incluyen fiebre, tos, dolor de cabeza, cansancio, diarrea, fatiga y neumonía. En algunos pacientes los síntomas pueden ser leves y, en otros, pueden agravarse en diversos sistemas como el respiratorio, cardiovascular o el nervioso central. Las personas mayores y con enfermedades crónico-degenerativas tienen mayor riesgo de complicaciones ${ }^{3-5}$.

El brote de COVID-19 comenzó en Wuhan (China) en diciembre de 2019, como una neumonía viral, y debido al número de casos, la gravedad de la enfermedad y su rápida expansión mundial, fue declarada por la Organización Mundial de la Salud, el 11 de marzo de 2019, como pandemia y emergencia de salud pública internacional ${ }^{6,7}$. 
Según el Centro de Recursos de Coronavirus de la Universidad Johns Hopkins, se han registrado más de cuatro millones de casos y más de 300 mil muertes, al 20 de mayo de $2020^{8}$. Asimismo, la implementación de medidas básicas de protección como el estricto confinamiento de personas que han sido o que no han sido potencialmente expuestas, se ha relacionado con síntomas de estrés, de estrés postraumático y de ansiedad, tanto en población general como en personal sanitario ${ }^{9-11}$.

Se han publicado dos revisiones acerca de los efectos sobre la salud mental de la COVID-199,12, pero en estas no se analizaron las tendencias en la investigación acerca de los síntomas y trastornos mentales. Brooks et al. ${ }^{9}$, analizaron el impacto psicológico de enfermedades como SARS, ébola, H1N1 y MERS, a partir de la revisión de 24 estudios indexados en Medline, PsycInfo y Web of Science. La revisión señala efectos psicológicos negativos, incluidos síntomas de estrés postraumático, "confusión"y enojo en pacientes diagnosticados y en población general en cuarentena. Sin embargo, los autores no incluyeron estudios relacionados específicamente con la COVID-19.

En la revisión de Chévance et al. ${ }^{12}$, se analizaron cartas al editor y artículos sobre pacientes hospitalizados con trastornos mentales en China, así como adultos mayores que tenían un alto riesgo de presentar trastornos psicológicos durante la pandemia de COVID-19. Los autores destacaron la necesidad de contar con más información acerca del efecto de la pandemia en personas con trastornos mentales diagnosticadas de COVID-19, bien sea de población general o de profesionales sanitarios.

Las revisiones anteriores adolecen de información acerca de las tendencias en la investigación sobre los síntomas y los trastornos mentales durante la pandemia de COVID-19. Dicha información es importante para complementar el conocimiento sobre las dificultades de salud mental asociadas a esta pandemia, y para aportar al establecimiento de estrategias de prevención de trastornos mentales. Conforme a lo anterior, el objetivo de esta revisión sistemática es describir las tendencias de investigación sobre los síntomas y trastornos mentales durante la pandemia de COVID-19.

\section{METODOLOGÍA}

\section{Tipo de estudio}

Esta revisión sistemática es un estudio ex-post-facto bibliométrico ${ }^{13}$; para realizarla se buscaron artículos publicados entre el 31 de diciembre de 2019 y el 3 de mayo de 2020 y se siguieron los lineamientos de la metodología PRISMA ${ }^{14}$.

\section{Unidades de análisis}

Se recuperaron artículos entre el 31 de diciembre 2019, fecha en la que la Organización Mundial de la Salud emitió una alerta por la infección por causa de la COVID-19, y el 3 de mayo de 2020, que dieran cuenta de las tendencias en investigación sobre síntomas de trastornos mentales y del comportamiento en tiempo de la epidemia por causa de esta afección, a través de los sistemas de información y bases de datos Pubmed, SCOPUS, EBSCO, psycINFO, ScienceDirect, Redalyc $\mathrm{y}$ Web of Science.

\section{Estrategia de búsqueda}

Las pesquisas se realizaron por dos autores de manera independiente, del 15 de abril al 3 de mayo de 2020, en los siete sistemas de información, por medio de una estrategia de búsqueda avanzada o búsqueda asistida, realizada en los campos "título" y "resumen". En el caso específico de REDALYC, esta búsqueda se llevó a cabo seleccionando "psicología" como área de conocimiento de las revistas.

Se implementaron los siguientes pasos: (a) definición de las palabras clave, tanto en inglés como en español, que se utilizarían en la búsqueda, (b) establecimiento de las combinaciones entre palabras clave necesarias para acceder a la mayor cantidad de información, (c) definición de la estrategia de búsqueda utilizada en los sistemas de información, y (d) establecimiento del protocolo de revisión y registro de resultados.

Se llevó a cabo un análisis deductivo categorial con base en los títulos y resúmenes de los artículos, a la luz de las siguientes palabras clave (Medical Subject Headings o $\mathrm{MeSH}$ ), usadas en cada búsqueda:

- Español: Enfermedad mental OR trastorno mental OR trastorno de ansiedad; ansiedad OR ira OR trastorno de ansiedad generalizada OR delirio OR ansiedad social o fobia social OR depresión OR trastorno depresivo OR trastorno bipolar OR trastorno de estrés postraumático OR trastorno de conducta alimentaria OR trastorno obsesivo compulsivo OR trastorno psicótico OR trastorno de pánico OR trastorno de personalidad OR intolerancia a la incertidumbre OR insomnio OR trastorno del sueño OR control de impulsos AND epidemia COVID-19

- Inglés: Mental illness OR mental disorders OR anxiety disorders $O R$ anxiety $O R$ anger $O R$ delirium $O R$ social phobia OR depression OR depressive disorder OR bipolar and related disorders $O R$ obsessive-compulsive disorder $O R$ panic disorder OR personality disorders OR sleep wake disorders OR sleep wake disorders AND COVID-19 epidemic. 


\section{Selección de artículos y extracción de datos}

Tres autores participaron en la selección y extracción de estudios, a través de reuniones de consenso para la inclusión de los artículos seleccionados. Los criterios de inclusión fueron establecidos a partir de aquellos del formato PICOS ${ }^{15}$ : (a) En la búsqueda y selección los artículos debían contener mínimo una de las palabras claves junto con la palabra clave "epidemia COVID-19" o "COVID-19 epidemic", buscando que dichos trabajos se refirieran a síntomas o trastornos mentales en tiempo de la epidemia por COVID-19; (b) los estudios elegidos debían ser artículos empíricos que contaran con calidad metodológica; (c) para sintetizar el alcance e impacto de las investigaciones, cada artículo seleccionado se reseñó conforme a los siguientes elementos: autores; país de origen, tipos de síntomas de trastornos mentales estudiados, fundamentos teóricos de la intervención, descripción de los participantes (rango, media de edad de los participantes, tamaño de la muestra) tipo de estudio, tipo de diseño, objetivo del estudio, comparadores, estadísticos utilizados, descripción de resultados, aportes o alcances $\mathrm{y}$ limitaciones.

No se consideraron criterios específicos de calidad de los artículos, debido a que el objetivo del presente trabajo fue realizar una revisión sistemática de la tendencia de investigación sobre síntomas de trastornos mentales durante la pandemia por COVID-19.

\section{RESULTADOS}

Se obtuvieron 99 artículos a partir de los sistemas de información, que contenían las palabras clave. De estos se eliminaron 55 registros por estar duplicados, y 28, porque no eran estudios empíricos sobre salud mental durante la pandemia. Por tanto, se revisaron y analizaron un total de 16 estudios según el protocolo establecido (ver Figura 1).

De las investigaciones seleccionadas, el $87.5 \%$ fue en China para evaluar el impacto psicológico que tuvo la cuarentena en la población general; así como en los trabajadores sanitarios que combatían la epidemia; en estudiantes universitarios y en trabajadores al retornar a sus labores después de confinamiento. El 12.5\% de los artículos fueron en población italiana general y en pacientes con enfermedad autoinmune que pasaron de atención hospitalaria a domiciliaria por la cuarentena. En general, los objetivos de los estudios comprendían identificar, analizar, evaluar y comparar los síntomas mentales y los efectos psicológicos como la ansiedad, la depresión, los trastornos del sueño-vigilia, el estrés, entre otros, en relación con la pandemia.
El tamaño de la muestra de los estudios osciló entre 20 y 7236 participantes, en su mayoría adolescentes y adultos. Solo una investigación incluyó niños, con una edad mínima de 12 años. Dos de las investigaciones tomaron en cuenta como fuente a trabajadores sanitarios, una evaluó a estudiantes universitarios y las restantes examinaron la población en general. El rango de edad de los participantes varió entre 12 y 80 años. El tipo de muestreo más utilizado fue el no probabilístico, por conveniencia y bola de nieve.

Con respecto a los estadísticos usados para los análisis, el $62.5 \%$ emplearon pruebas paramétricas, siendo la prueba $\mathrm{t}$ de student para muestras independientes la que más se empleó, en un $21.8 \%$ de los estudios, seguida por la regresión lineal múltiple, con un 15.6\%, análisis de invarianza (ANOVA), con un $9.3 \%$ y en cuarto lugar el coeficiente de correlación de Pearson, con 6,25\%. El $37,5 \%$ restante emplearon pruebas no paramétricas,

Figura 1. Diagrama de flujo de la selección de los estudios de tendencias en la investigación en trastornos mentales y del comportamiento durante la epidemia por COVID-19.
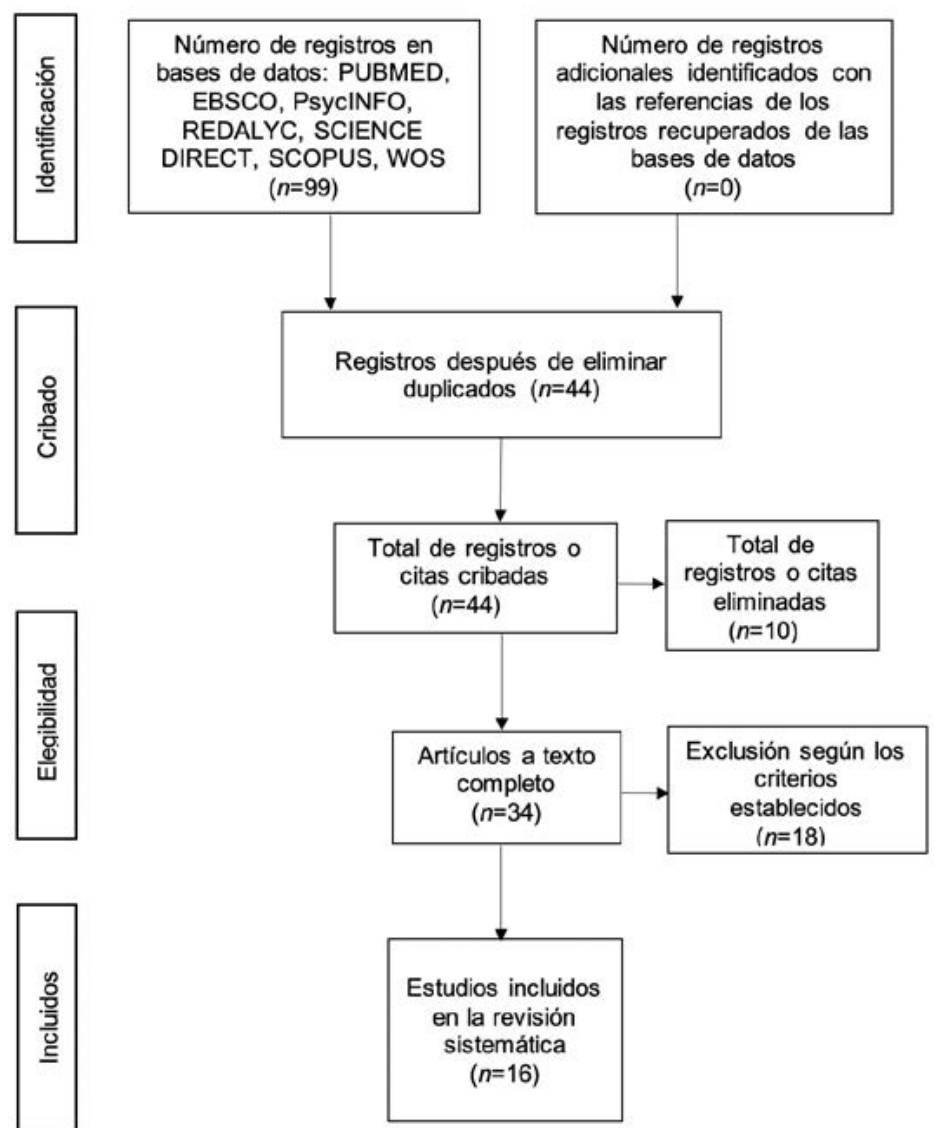
donde el estadístico Chi-cuadrado de Pearson fue el más usado, en un $25 \%$ de las investigaciones, seguido por la $U$ de Mann Whitney, la prueba de Fisher y de Spearman, cada uno en un $3.12 \%$ de los estudios.

Se utilizaron 35 tipos diferentes de cuestionarios, escalas y listas para recolectar los datos en los 16 estudios seleccionados. Los instrumentos más empleados fueron: (a) La escala de depresión, ansiedad y estrés DASS- $21^{16}$, que mide síntomas de estas tres condiciones con base en la triada psicopatológica de Clark y Watson (1991a), utilizado en el 11.4\% de los estudios; (b) la escala de trastorno de ansiedad generalidad ${ }^{17}$, que estudia los síntomas centrales de este trastorno y su frecuencia en las últimas dos semanas, el cual se usó en el 8.5\% de los estudios; (c) la escala de autoevaluación de ansiedad $(\mathrm{SAS})^{18}$, que permite determinar los niveles de gravedad de la ansiedad, en un $8.5 \%$ de las investigaciones; (d) el índice de calidad del sueño de Pittsburgh ${ }^{19}$, que evalúa la duración y la calidad del sueño, utilizado también en un $8.5 \%$ de los estudios, y (e) la escala de impacto de eventos revisada $(\mathrm{IES}-\mathrm{R})^{20}$, la cual se emplea para determinar el alcance del impacto psicológico después de la exposición a una situación de emergencia en salud pública, implementada en un 5.7\% de las investigaciones. En todos los artículos se resalta que los instrumentos han sido validados y se han aplicado ampliamente en las poblaciones evaluadas.

Los artículos seleccionados examinaron los siguientes 14 síntomas o trastornos mentales: ansiedad (28.8\%), depresión (23\%), estrés (15.3\%), trastorno del sueño-vigilia (9.6\%), trastorno de estrés postraumático (5.7\%), trastornos relacionados con sustancias y trastornos adictivos (1.9\%), somatización (1.9\%), obsesivo compulsivo (1.9\%), sensibilidad interpersonal (1.9\%) hostilidad (1.9\%), ansiedad fóbica (1.9\%), ideación paranoide (1.9\%), psicoticismo (1.9\%) y suicidio (1.9\%) (ver Tabla 1).

La ansiedad, que fue la característica más estudiada (en el 28.8\% de los estudios), evidenció una prevalencia de entre el $8.3 \%$ y el 29\%. Algunos de los estudios examinaron la ansiedad en tres niveles (leve, moderado y severo), particularmente, los estudios que utilizaron la escala de autoevaluación de ansiedad (SAS) y la escala de trastorno de ansiedad generalidad (GAD-7), donde la ansiedad leve fue la más predominante, con una prevalencia entre el $2.1 \%$ y el $21.3 \%$; la moderada, con una prevalencia entre el 2.1\% y el 6\%; y la ansiedad grave, con una prevalencia de entre el $0.9 \%$ y el 12.9\% (ver Tabla 2).

Además, la prevalencia de ansiedad fue significativamente mayor en el grupo de personas menores de 35 años y, en algunas investigaciones, el sexo femenino se asoció con mayores puntuaciones en esta condición. Finalmente, las personas que vivían solas, solteras, divorciadas o viudas presentaban puntuaciones mayores de ansiedad ${ }^{21-25}$. Tan et $a_{1}{ }^{26}$, encontraron que las personas que sentían estar en riesgo al regresar a sus puestos trabajo obtenían puntuaciones mayores en ansiedad. Finalmente, entre los trabajadores de la salud, aquellos que tenían menos experiencia presentaban mayores índices de ansiedad ${ }^{27,28}$.

El cuanto al segundo trastorno más estudiado, la depresión (en un 23\% de los estudios), algunos trabajos informaron prevalencias de depresión leve entre el 8.3\% y el $13.8 \%$; moderada, entre el $5.2 \%$ y el $12.2 \%$; y grave, entre el $1.1 \%$ y el $4.3 \%^{23,29}$. Los resultados de estas investigaciones señalan que las puntuaciones altas en las escalas para depresión se asocian con el consumo de sustancias psicoactivas (especialmente con el alcohol), el bajo nivel de estudios académicos, la pérdida económica, entre las personas menores de 35 años, el regreso al lugar de trabajo, el poco autocuidado y el estado de salud autopercibido $23,26,29-31$.

En relación con el estrés, el tercer tipo de síntoma mental más estudiado (15.3\%), en algunas investigaciones se encontró que las personas que estaban expuestas a la cuarentena y que percibieron el regreso al trabajo como un peligro para la salud o tenían una percepción negativa de su estado de salud, tuvieron puntuaciones de estrés significativamente más altas ${ }^{26,29}$. De otro lado, los participantes que confiaban en las capacidades de los médicos y del sistema de salud para diagnosticar o reconocer el COVID-19 obtuvieron puntuaciones más bajas en esta variable ${ }^{24}$. Los patrones de sueño se vieron afectados cuando las personas informaban síntomas de estrés $^{32}$. De igual manera, quienes presentaba problemas económicos puntuaban más alto en esta variable, lo que afectaba la calidad del sueño ${ }^{33}$.

\section{DISCUSIÓN}

El objetivo de la presente revisión fue analizar las tendencias de investigación sobre los síntomas y trastornos mentales durante la epidemia de la COVID-19, a partir de los sistemas de información Pubmed, SCOPUS, EBSCO, PsycInfo, ScienceDirect, Redalyc y Web of Science. Se analizaron 16 artículos publicados a partir del año 2020; 14 de ellos realizados en China en población general, profesionales sanitarios, estudiantes universitarios y trabajadores que retomaron sus labores después del confinamiento, mientras que dos estudios se realizaron en Italia en población general y pacientes con enfermedad autoinmune. Todos los artículos seleccionados analizaron, evaluaron y compararon los efectos psicológicos relacionados con síntomas de ansiedad, depresión, trastornos del sueño-vigilia y estrés asociados con las diferentes etapas de la pandemia de la COVID-19.

Con respecto a las características de los participantes, la mayoría de los estudios se realizó con adolescentes y 


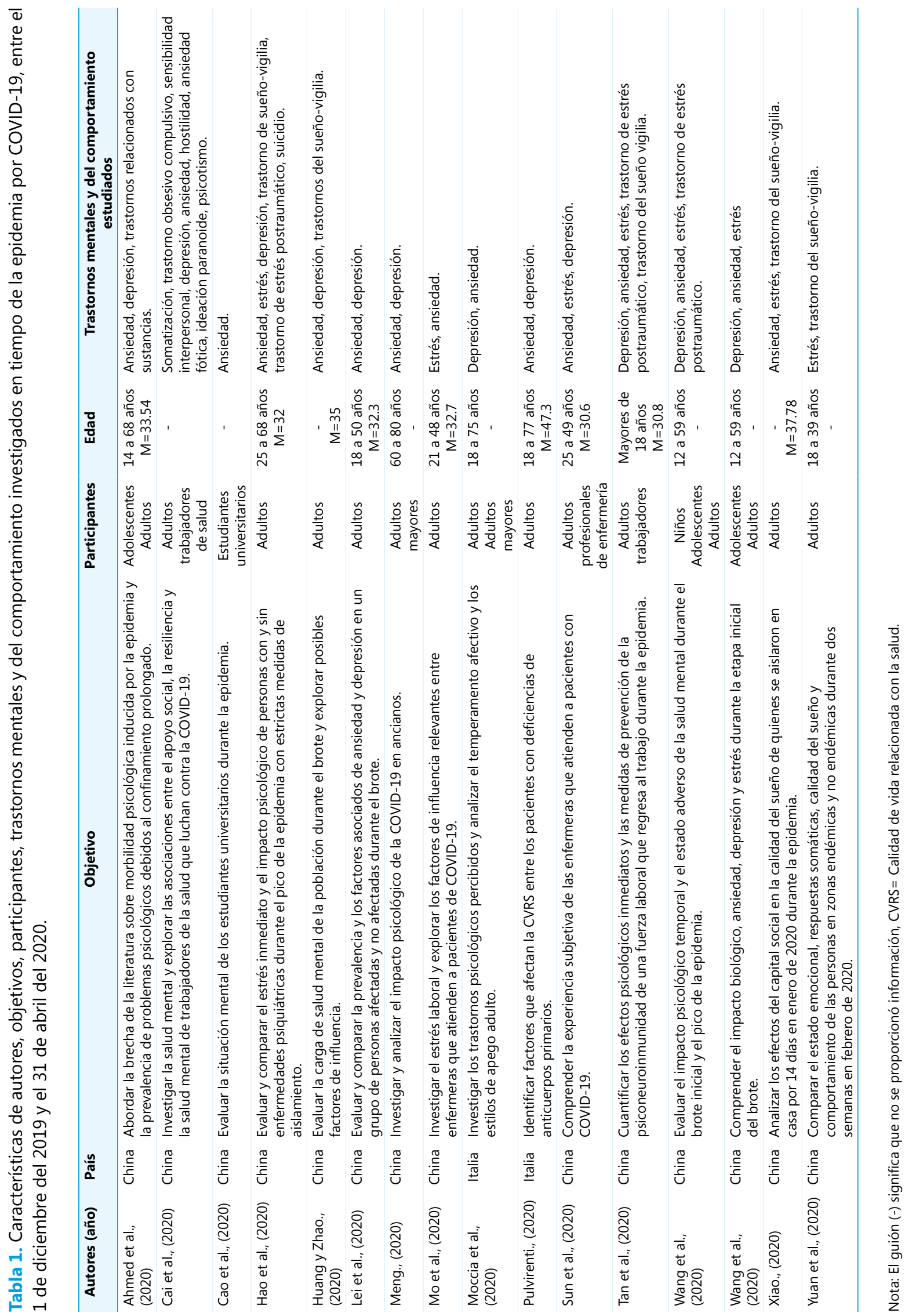




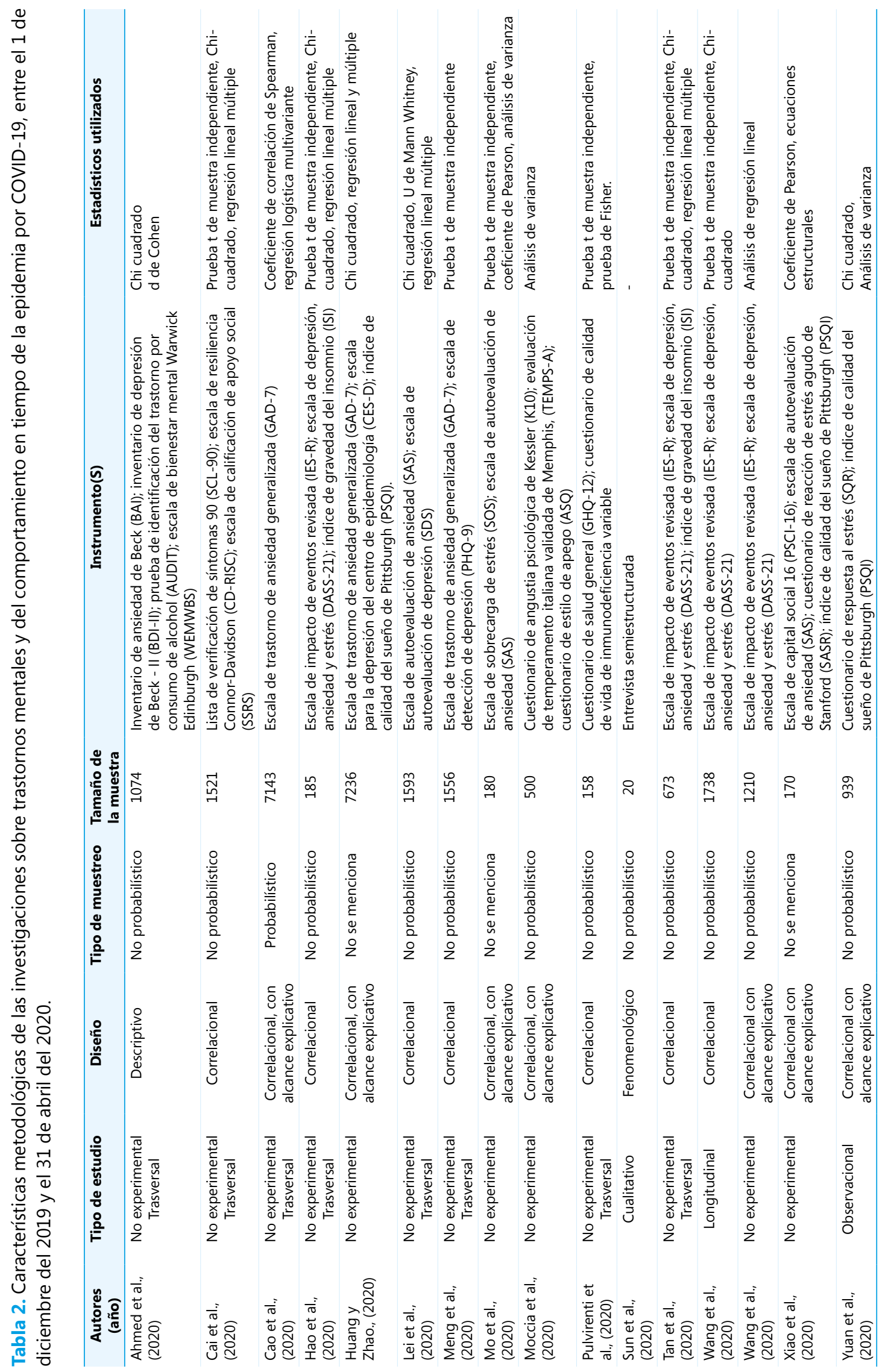


adultos de la población general, mientras que otros se hicieron con personas con enfermedad autoinmune y personal sanitario, todos con edades entre los 12 y 80 años. Por consiguiente, un área de oportunidad para futuras investigaciones es el estudio de los trastornos mentales durante la pandemia en niños, en pacientes psiquiátricos, internos de cárceles, así como en familiares y cuidadores de personas con la COVID-19 12,34 .

Referente a los instrumentos de medición utilizados para recolectar datos, estos correspondían a pruebas de autorreporte y tipo screening, validadas en las poblaciones de estudio, lo que contribuye a disminuir el error de medición, y aumenta la presión y exactitud en la medición de las variables. Este tipo de pruebas son utilizadas para hacer tamizaje y permiten conocer el comportamiento de una variable psicológica en una población general, examinado diversos síntomas psicológicos. Sin embargo, a pesar del uso de este tipo de instrumentos, la principal limitación de estos estudios fue que no contaron con información de estos síntomas o trastornos de manera previa a la pandemia, por lo que no se puede establecer si estos aparecieron con la epidemia o en la cuarentena, o si dicha coyuntura los exacerbó.

Para los análisis estadísticos, la salud mental cuenta con un gran número de métodos que permiten responder las diferentes preguntas de investigación que se formulan en sus disciplinas. Estos métodos se pueden dividir en paramétricos y no paramétricos. Los primeros hacen suposiciones de la naturaleza de la población a partir de una muestra, mientras que los segundos no hacen suposiciones tan severas acerca de los parámetros poblacionales. En esta investigación se encontró que los estadísticos más utilizados son los paramétricos, lo que supone que los datos se obtuvieron de una población distribuida normalmente o que tenían la misma varianza o distribución. Sin embargo, pocos artículos señalan si se cumplieron los supuestos de normalidad y homogeneidad de las varianzas, y menos los que indican el cumplimiento de aleatoriedad de la muestra (muestreo aleatorio). Esta condición puede afectar la precisión y potencia estadísticas de los estudios, al incurrir en posibles sesgos y errores al aceptar o rechazar las hipótesis planteadas.

Para el caso específico de los síntomas y trastornos mentales, los estudios examinaron 14, siendo los más frecuentes la ansiedad y la depresión, con un $28 \%$ y $23 \%$ respectivamente, lo que indica un interés mayor hacia el estudio de tales dificultades durante la pandemia de COVID-19, mientras que las dificultades menos estudiadas fueron los trastornos de sustancias y adictivos, somatización, síntomas obsesivo-compulsivos, sensibilidad interpersonal, hostilidad, ansiedad fóbica, ideación paranoide, psicoticismo, estrés, trastorno de estrés postraumático, trastorno del sueño-vigilia y suicidio. $\mathrm{Al}$ respecto, Brooks et al. ${ }^{12}$, refieren que la cuarentena de diferentes enfermedades como SARS, ébola, H1N1 y MERS, se ha relacionado con efectos psicológicos negativos en el estado de ánimo y la ansiedad.

La prevalencia de la ansiedad osciló entre el 8.3\% y el $29 \%$. Al contrastar estas cifras con estadísticas previas, se evidencia un posible aumento de dicha característica. Por ejemplo, en China, la prevalencia de ansiedad en la población general, antes de la pandemia, oscilaba entre el $3.1 \%$ y el $24 \%{ }^{35,36}$, y en Italia, entre el $5 \%$ y el $10.3 \%{ }^{37,38}$. Además, la prevalencia de ansiedad fue mayor entre las personas menores de 35 años y del sexo femenino, lo que es consistente con las diferencias por sexo ya conocidas. Arenas y Puigcerver ${ }^{39}$ señalan, al respecto, que durante el curso de la vida, el 17,5\% de las mujeres y el 9,5\% de los hombres sufrirán algún síntoma de trastornos de ansiedad, y que las mujeres en edad reproductiva pueden tener entre dos y tres veces mayor probabilidad de presentar estos síntomas que los hombres.

Los hallazgos de los estudios también señalan que hay relación entre los síntomas de trastornos depresivos y estrés, con el consumo de sustancias psicoactivas, el bajo rendimiento académico, la pérdida de trabajo, la calidad del sueño, el pobre autocuidado y la percepción de salud frágil durante la pandemia, lo que coincide con estudios sobre el SARS ${ }^{40-42}$.

La principal limitación de nuestro estudio yace en la barrera idiomática, puesto que la búsqueda se realizó solo español e inglés, entre el 31 de diciembre de 2019 y el 3 de mayo de 2020, por lo que se recomienda que, en futuras investigaciones, se consideren artículos en todos los idiomas y posteriores a las fechas de búsqueda, debido a que muchos países se encuentran aún en confinamiento y la pandemia de la COVID-19 no ha culminado.

Para concluir, las investigaciones sobre síntomas de trastornos mentales durante la pandemia por COVID-19 aún son escasas, y más en Iberoamérica, en donde es poca o nula la producción científica sobre el tema. Además, se evidencia la falta de estudios que analicen programas de intervención, prevención y promoción de la salud mental, que busquen disminuir el impacto psicológico de la pandemia, por lo que se recomienda que se realicen investigaciones de intervención, que tengan en cuenta factores sociales y culturales de las personas en confinamiento, específicamente para niños, adolescentes, adultos, pacientes y personal sanitario ${ }^{43}$. También, se requieren estudios de seguimiento de los síntomas y trastornos mentales en diferentes poblaciones. 


\section{REFERENCIAS}

1. Kirkcaldy RD, King BA, Brooks JT. COVID-19 and postinfection immunity: Limited evidence, many remaining questions. JAMA. 2020 (en prensa); doi: 10.1001/jama.2020.7869.

2. OMS [Internet]. Brote de enfermedad por coronavirus COVID-19. [citado el 20 de mayo de 2020]. Disponible en: https://www.who.int/emergencies/diseases/novel-coronavirus-2019

3. Rothan HA, Byrareddy SN. The epidemiology and pathogenesis of coronavirus disease (COVID-19) outbreak. J Autoimmun. 2020 (en prensa); doi: 10.1016/j.jaut.2020.102433.

4. Xu Z, Shi L, Wang Y, Zhang J, Huang L, Zhang C, et al. Pathological findings of COVID-19 associated with acute respiratory distress syndrome. Lancet Respir Med. 2020; 8(4):420-2.

5. Zu ZY, Jiang MD, Xu PP, Chen W, Ni QQ, Lu GM, et al. Coronavirus disease 2019 (COVID-19): A perspective from China. Radiology. 2020 (en prensa); doi: 10.1148/radiol.2020200490.

6. CDC [Internet]. Coronavirus (COVID-19) [citado el 20 de mayo de 2020]. Disponible en: https:// www.cdc.gov/coronavirus/2019-ncov/index.html

7. OMS [Internet]. Time line COVID-19 [citado el 20 de mayo de 2020]. Disponible en: https:// www.who.int/news-room/detail/27-04-2020-who-timeline---covid-19

8. Center for Systems Science and Engineering [Internet]. Coronavirus resource center, COVID-19 Dashboard by the Center for Systems Science and Engineering (CSSE) [citado el 20 de mayo de 2020]. Disponible en: https://coronavirus.jhu.edu/map.html

9. Brooks SK, Webster RK, Smith LE, Woodland L, Wessely S, Greenberg N. The psychological impact of quarantine and how to reduce it: Rapid review of the evidence. Lancet. 2020; 395(10227):912-20.

10. De Girolamo G, Cerveri G, Clerici M, Monzani E, Spinogatti F, Starace F, et al. Mental health in the Coronavirus disease 2019 emergency - the Italian response. JAMA Psychiatry. 2020 (en prensa); doi: 10.1001/jamapsychiatry.2020.1276

11. Rubin GJ, Wessely S. The psychological effects of quarantining a city. BMJ 2020; 368:m313.

12. Chevance A, Gourion D, Hoertel N. Assurer les soins aux patients souffrant de troubles psychiques en France pendant l'épidémie à SARS-CoV-2. Encéphale. 2020; S0013-7006(20)30064-6.

13. Montero I, León OG. A guide for naming research studies in psychology. Int J Clin Health Psychol. 2007; 7:847-62.

14. Liberati A, Altman DG, Tetzlaff J, Mulrow C, Gotzsche PC, loannidis JP, et al. The PRISMA statement for reporting systematic reviews and meta-analyses of studies that evaluate health care interventions: Explanation and elaboration. Plos Med 2009; 6(7): e1000100.

15. Perestelo-Pérez L. Standards on how to develop and report systematic reviews in psychology and health. Int J Clin Health Psychol. 2013; 13:49-57.

16. Norton PJ. Depression Anxiety and Stress Scales (DASS-21): Psychometric analysis across four racial groups. Anxiety Stress Coping. 2007; 20(3):253-65.

17. Spitzer RL, Kroenke K, Williams JBW, Löwe B. A brief measure for assessing generalized anxiety disorder: The GAD-7. Arch Intern Med. 2006; 166(10):1092-7.

18. Olatunji BO, Deacon BJ, Abramowitz JS, Tolin DF. Dimensionality of somatic complaints: Factor structure and psychometric properties of the Self-Rating Anxiety Scale. J Anxiety Disord. 2006; 20(5):543-61.

19. Backhaus J, Jughanns K, Broocks A, Riemann D, Hohagen F. Test-retest reliability and validity of the Pittsburgh Sleep Quality Index in primary insomnia. J Psychosom Res. 2002; 53:737-40.

20. Wu KK, Chan KS. The development of the Chinese version of Impact of Event Scale--Revised (CIES-R). Soc Psychiatry Psychiatr Epidemiol. 2003; 38(2):94-8.

21. Ahmed MZ, Ahmed O, Aibao Z, Hanbin S, Siyu L, Ahmad A. Epidemic of COVID-19 in China and Associated Psychological Problems. Asian J Psychiatr 2020; 51:102092.

22. Cao W, Fang Z, Hou G, Han M, Xu X, Dong J, et al. The psychological impact of the COVID-19 epidemic on college students in China. Psychiatry Res. 2020; 287:112934.

23. Lei L, Huang X, Zhang S, Yang J, Yang L, Xu M. Comparison of prevalence and associated factors of anxiety and depression among people affected by versus people unaffected by quarantine during the COVID-19 epidemic in Southwestern China. Med Sci Monit. 2020; 26: e924609.

24. Wang C, Pan R, Wan $X$, Tan $Y, X u$ L, Mclntyre RS, et al. A longitudinal study on the mental health of general population during the COVID-19 epidemic in China. Brain Behav Immun. 2020; S0889-1591(20)30511-0.

25. Huang $Y$, Zhao N. Generalized anxiety disorder, depressive symptoms and sleep quality during COVID-19 outbreak in China: A web-based cross-sectional survey. Psychiatry Res. 2020; 288:112954.

26. Tan $W$, Hao F, Mclntyre RS, Jiang $L$, Jiang $X$, Zhang $L$, et al. Is returning to work during the COVID-19 pandemic stressful? A study on immediate mental health status and psychoneuroimmunity prevention measures of Chinese Workforce. Brain Behav Immun. 2020; S0889-1591(20)30603-6.

27. Mo Y, Deng L, Zhang L, Lang Q, Liao C, Wang N, et al. Work stress among Chinese nurses to support Wuhan for fighting against the COVID-19 epidemic. J Nurs Manag. 2020; 7:10.1111/ jonm.13014. 
28. Cai W, Lian B, Song X, Hou T, Deng G, Li H. A cross-sectional study on mental health among health care workers during the outbreak of Corona Virus Disease 2019. Asian J Psychiatr. 2020; 51:102111.

29. Wang C, Pan R, Wan X, Tan Y, Xu L, Ho CS, et al. Immediate psychological responses and associated factors during the initial stage of the 2019 Coronavirus disease (COVID-19) epidemic among the general population in China. Int J Environ Res Public Health. 2020; 17(5):1729.

30. Meng H, Xu Y, Dai J, Zhang Y, Liu B, Yang H. The psychological effect of COVID-19 on the elderly in China. Psychiatry Res. 2020; 289:112983.

31. Hao F, Tan W, Jiang L, Zhang L, Zhao X, Zou Y, et al. Do psychiatric patients experience more psychiatric symptoms during COVID-19 pandemic and lockdown? A case-control study with service and research implications for immunopsychiatry. Brain Behav Immun. 2020; S08891591(20)30626-7.

32. Sun $N$, Wei $L$, Shi S, Jiao $D$, Song $R$, Ma $L$, et al. A qualitative study on the psychological experience of caregivers of COVID-19 patients. Am J Infect Control. 2020; 48(6):592-8.

33. Xiao H, Zhang Y, Kong D, Li S, Yang N. Social capital and sleep quality in individuals who selfisolated for 14 days during the Coronavirus disease 2019 (COVID-19) outbreak in january 2020 in China. Med Sci Monit. 2020; 26:e923921.

34. Holmes EA, O'Connor RC, Perry VH, Tracey I, Wessely S, Arseneault L, et al. Multidisciplinary research priorities for the COVID-19 pandemic: A call for action for mental health science. Lancet Psychiatry. 2020; 7(6):547-60.

35. Guo X, Meng Z, Huang G, Fan J, Zhou W, Ling W, et al. Meta-analysis of the prevalence of anxiety disorders in mainland China from 2000 to 2015. Sci Rep. 2016; 6:28033.

36. Yu W, Singh SS, Calhoun S, Zhang H, Zhao X, Yang F. Generalized anxiety disorder in urban China: Prevalence, awareness, and disease burden. J Affect Disord. 2018; 234:89-96.

37. De Girolamo G, Polidori G, Morosini P, Scarpino V, Reda V, Serra G, et al. Prevalence of common mental disorders in Italy: results from the European Study of the Epidemiology of Mental Disorders (ESEMeD). Soc Psychiatry Psychiatr Epidemiol. 2006; 41(11):853-61.

38. OMS [Internet]. Depresión y otros trastornos mentales comunes. Estimaciones de salud global [citado el 20 de mayo de 2020]. Disponible en: https://apps.who.int/iris/bitstream/ handle/10665/254610/WHO-MSD-MER-2017.2-eng.pdf

39. Ahmed MZ, Ahmed O, Aibao Z, Hanbin S, Siyu L, Ahmad A. Epidemic of COVID-19 in China and associated psychological problems. Asian J Psychiatr. 2020; 51:102092.

40. Chua SE, Cheung V, McAlonan GM, Cheung C, Wong JWS, Cheung EP, et al. Stress and psychological impact on SARS patients during the outbreak. Can J Psychiatry. 2004; 49(6):38590.

41. Lee AM, Wong JG, McAlonan GM, Cheung V, Cheung C, Sham PC, et al. Stress and psychological distress among SARS survivors 1 year after the outbreak. Can J Psychiatry. 2007; 52(4):233-40.

42. Wu KK, Chan SK, Ma TM. Posttraumatic stress, anxiety, and depression in survivors of severe acute respiratory syndrome (SARS). J Trauma Stress. 2005; 18(1):39-42.

43. Urzúa A, Vera-Villarroel P, Caqueo-Urízar A, Polanco-Carrasco R. La psicología en la prevención y manejo del COVID-19. Aportes desde la evidencia inicial. Terapia Psicológica. 2020; 38(1):103-8. 